\title{
Retroperitoneal angioleiomyomatosis
}

\author{
Luz Angela Torres-de la Roche ${ }^{1}\left({ }^{\oplus} \cdot\right.$ Rajesh Devassy $^{1,2} \oplus \cdot$ Ghaith Makhlouf $^{2} \cdot$ Johannes San Juan $^{2}$. \\ Jennifer Eidswick ${ }^{1} \cdot$ Rudy Leon De Wilde $^{1}$ (I)
}

Received: 11 June 2020 / Accepted: 20 November 2020 / Published online: 23 December 2020

(C) The Author(s) 2020

\begin{abstract}
Retroperitoneal intravenous leiomyomatosis is a rare benign tumor that can spread through veins carrying significant morbidity. The challenge of its management lies within the complexity of completely excising the tumor, which if carried out improperly can result in neurological or vascular complications requiring complex reparative surgeries. Here we present the successful resection of a retroperitoneal angio-leiomyoma by combining laparoscopic route, micro-surgical techniques and modern endoscopic tools.
\end{abstract}

Keywords Intravenous leiomyomatosis · Laparoscopy $\cdot$ Minimally invasive surgery $\cdot$ Angiomyoma $\cdot$ Immunomarkers

\section{Introduction}

Intravenous leiomyomata (IVL) are rare benign vascular neoplasms characterized by intraluminal growth of smooth muscle into either venous or lymphatic vessels outside the limits of myoma. Uterine IVL can extend through the veins, with inferior vena cava extension or pulmonary and heart metastasis, carrying significant morbidity [1-3]. Retroperitoneal development is another unusual growth pattern of uterine leiomyomas and especially of IVL. Retroperitoneal neural soft tissue tumors or angio-sarcoma lesions should

Luz Angela Torres-de la Roche, MD University Hospital for Gynecology, Pius Hospital, Carl von Ossietzky University, Oldenburg, Germany; Rajesh Devassy is M.S., D.M.I.C. University Hospital for Gynecology, Pius Hospital, Carl von Ossietzky University, Oldenburg, Germany and Dubai London Clinic and Specialty Hospital, Dubai; Rudy Leon De Wilde, MD, PhD University Hospital for Gynecology, Pius Hospital, Carl von Ossietzky University, Oldenburg, Germany.

Electronic supplementary material The online version of this article (https://doi.org/10.1007/s13224-020-01404-7) contains supplementary material, which is available to authorized users.

Rajesh Devassy

rajeshdevassy@gmail.com

1 University Hospital for Gynecology, Pius Hospital, Carl von Ossietzky University, Oldenburg, Germany

2 Department of Obstetrics and Gynecology, Dubai London Clinic and Speciality Hospital, Jumeirah St - Umm Suqeim-2, Dubai, United Arab Emirates be considered as differential diagnosis [2]. With regard to their pathologic origin, it is unclear whether these retroperitoneal lesions represent metastatic or synchronous primary lesions and whether they arise from hormonally sensitive smooth muscle elements. In immunohistochemical studies, IVL tumors have been reported to express different cell markers (p-16, or Cyclin 1 and present CD34 and D2-40 and cytoplasmic phosphorylated-Rb CD31,) and smooth muscle markers (Desmin and SMA). Recent molecular cytogenetic studies describe a monoclonal proliferation with genetic alterations usually seen in mesenchymal tumors, mainly $1 \mathrm{p}$, $22 q, 2 q, 1 q, 13 q$ and $14 q$ aberrations, supporting the theory that postulates IVL arise from intravascular projections of uterine myoma $[1,4]$. Most of the patients with an IVL present in the 5th and 6th decades of life with symptoms related to tumor localization. Surgical resection is often curative, but recurrences have occasionally been reported $[3,4]$. The challenge of IVL lies within the complexity of diagnosis and completely excising the tumor, which if carried out improperly may result in neurological or vascular complications requiring complex reparative surgeries.

\section{Case presentation}

A 35 year-old patient with two children underwent laparoscopic retroperitoneal pelvic tumor excision at the Dubai London Clinic and Speciality Hospital. The patient presented with chronic left leg pain for many years. At 
admission, ultrasound examination and MRT image findings were described as a mucinous cystadenoma of the left ovary $(9.2 \times 5.6 \mathrm{~cm}$ with relative irregular outline and filling the pouch of Douglas), with the differential diagnosis of a broad ligament leiomyoma with intrinsic tumor degeneration and necrotic features. By combining laparoscopic route, in-bag morcellation and micro-surgical technics, it was possible to ensure complete resection of the retroperitoneal tumor without intraoperative vascular or neural complications (Figs. 1, 2, 3, 4, 5 and 6; Ligasure TM, Sonicision TM, Endo Clip TM, Endo Catch TM, Veriset TM are Registered trade marks of Medtronic). At histological examination, the cytology of peritoneal fluid showed mild subacute inflammation with activated mesothelial cells, suggestive of exudative peritoneal effusion. Sections of the soft tissue neoplasm consisted of cellular sheets and irregular fascicles spindled cells with formation of glomeruloid architecture and epithelioid features. The lesion demonstrated numerous anastomosing structures resembling blood vessels covered by endothelium-like cells embedded in fibrous stroma. No evidence of malignancy or tumor necrosis was reported. The tumor was positive for immunomarkers CD31, CD34, D2-40, CD68, $\mathrm{Ki}-67$ (1-2\% positive nuclear staining) as well for smooth muscle markers SMA (Smooth muscle actin) and Desmine.
Fig. 1 Outline of the retroperitoneal mass upon laparoscopy

Fig. 2 Releasing superiorthe left external Iliac artery and Obturator with Ligasure ${ }^{\mathrm{TM}}$ lateral border of the tumor from
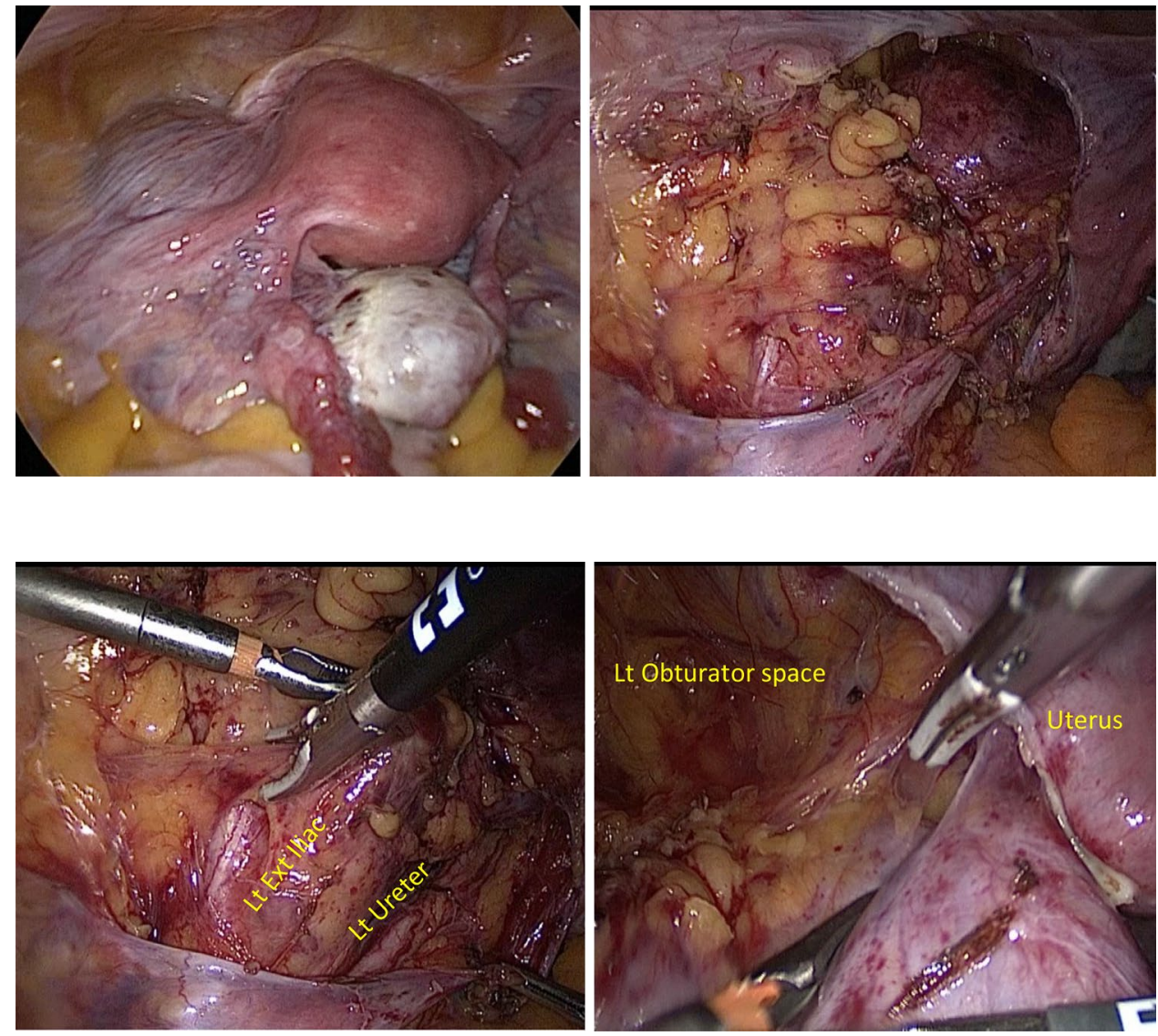

Fig. 3 Secluding the cephalic vascular attachments anterior to the external Iliac vessels possible branches of anterior division of IIA and/or tributaries of Iliolumbar artery
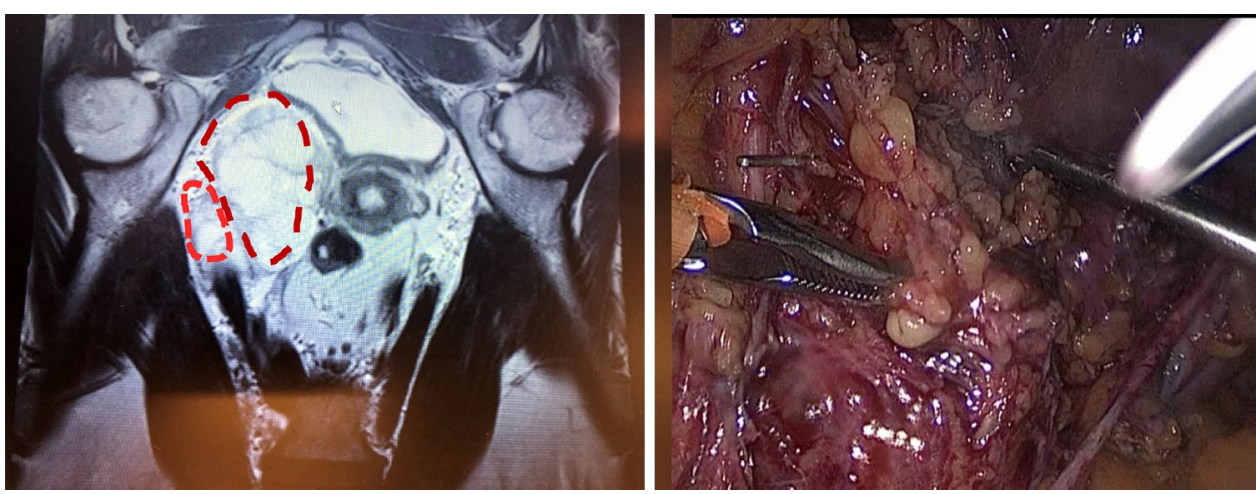
Fig. 4 Isolation and vascular clipping with Endo Clip TM of the branches supplying the tumor from uterine artery, medially isolated ureter is well appreciated
Fig. 5 Medial attachments of vascular branches arising out of the external iliac divided with Ligasure TM in between the vascular Endo Clip TM
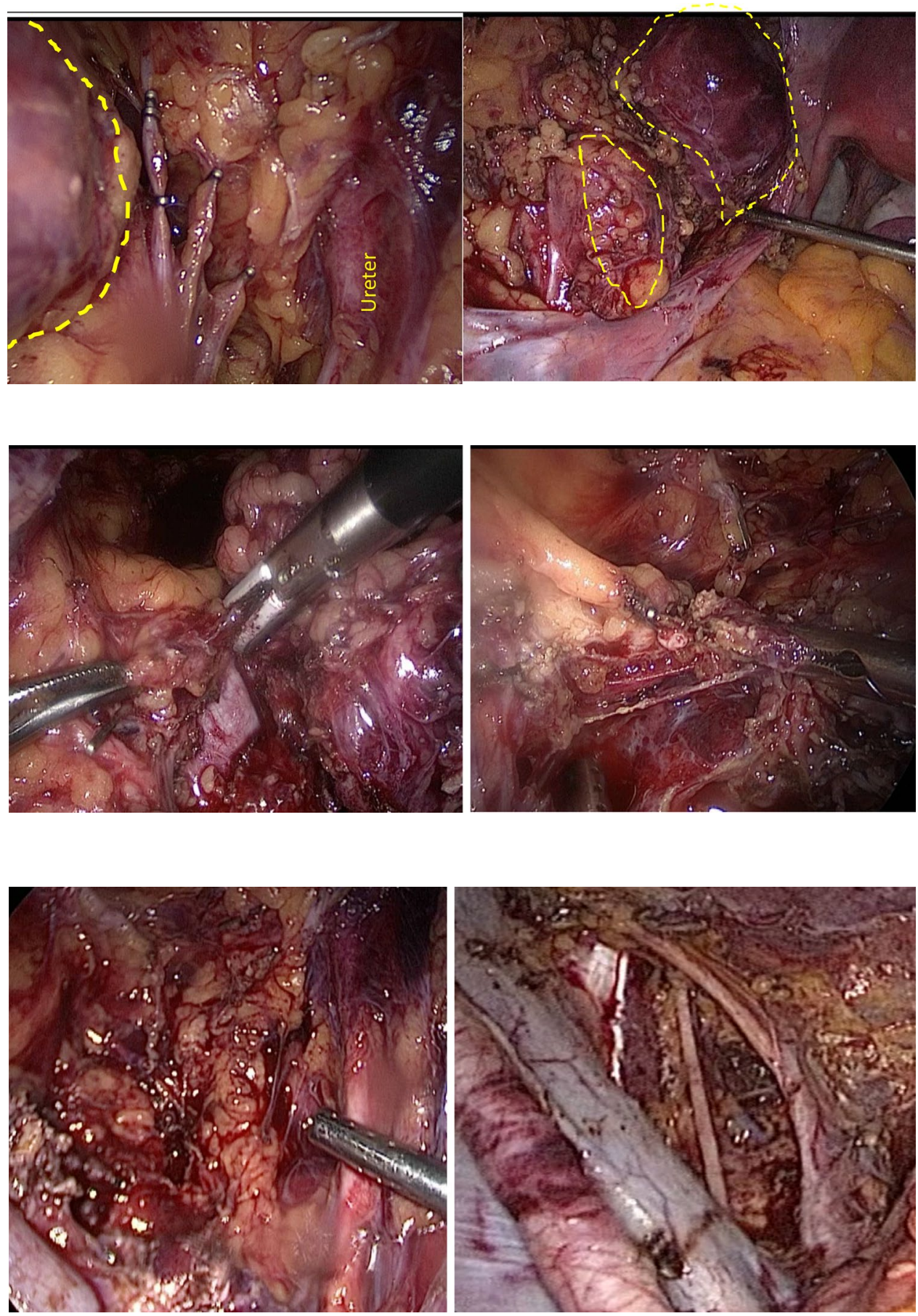

Fig. 6 Final hemostasis achieved confirmed in submersed LRS with hemostatic and adhesion barrier Veriset ${ }^{\mathrm{TM}}$ matrix patch
HHV-8, S100, Synaptophysin and Calretinin were negative. The postoperative course was uneventful.

\section{Discussion}

Multiple leiomyomatous masses are usually seen in the pelvic retroperitoneum in postmenopausal women with a concurrent uterine leiomyoma or a history of uterine leiomyoma. Rarely, the tumor exhibits smooth muscle proliferation within the vascular and lymphatic spaces of the myometrium, known as IVL, which in 30-80\% of cases propagates through its adjacent venous structures beyond the pelvis $[3,4]$. Affected patients may be asymptomatic or, if the tumor is limited to the pelvis, they may present with vaginal bleeding, pelvic or abdominal pain. In metastasizing disease, fatigue, ascites, peripheral edema, dyspnea, syncope, congestive heart failure and deep vein thrombosis may 
be present. When IVL presents as a retroperitoneal mass in the proximity of nerves, half of patients seek medical help due to chronic pelvic pain [1-4]. In contrast, we present a case of IVL diagnosed in a young patient (35 years-old) who, furthermore, presented with chronic leg pain not usually described in the literature.

IVL are not usually diagnosed before surgery. At simple ultrasound imaging, IVL are not easy to distinguish; at colorduplex examination, IVL presents as a heterogeneous vascular mass with high resistance. On axial contrast-enhanced CT-images, lesions resemble a 'sieve' and, on 3D volume rendered CT-images, a 'luffa sponge'. MRI imaging tumors can be seen as hypertensive and isointensive to skeletal muscle [5]. In this case, it was not possible to suspect the vascular anomaly of the tumor because no vascular Doppler was performed and at MRI the tumor was described as an ovarian mass (mucinous cystadenoma). Therefore, the diagnosis was made postoperatively by means of histopathology and cell markers examination. As reported in previous studies [1], the tumor was positive for immunomarkers CD31, CD34, D2-40, CD68, Ki-67 (1-2\% positive nuclear staining) as well for smooth muscle markers SMA and Desmine.

Surgery is the treatment of choice and is critical to assuring complete IVL resection to reduce recurrences. Percutaneous embolization could be used to reduce the size and vascularization of the tumor prior to surgical excision [3,4]. Despite the excellent prognosis, long-term imaging followup of patients is recommended. If any tumor is left in pelvic or affected veins, a bilateral oophorectomy should be considered for premenopausal women, since estrogen stimulates tumor growth [2,4]. In our case, a minimally invasive approach, by combining laparoscopic route, in-bag morcellation and micro-surgical technics, facilitated the complete tumor resection without complications, opening a feasible and safe avenue for the management of this type of tumor.

\section{Conclusion}

Retroperitoneal angio-leiomyoma is an extremely rare and yet a benign tumor. The complexity of its surgical treatment lies in its localization nearby to vascular and nerve structures and the high-risk of perioperative complications. However, successful resection of a giant retroperitoneal angio-leiomyoma was achieved by combining laparoscopic route, microsurgical techniques and modern endoscopic tools, including vascular clips, ultrasonic dissectors and in-bag morcellation. We encourage surgeons to offer this approach to their future patients.

Acknowledgments The authors thank Mrs. Jennifer Eidswick for the help in the English editing of the manuscript.
Authors' Contribution “Conceptualization, LATR, RD and RLDW; formal analysis, writing - original draft preparation, all authors; writing-review and editing, LATR and RD; supervision, RLDW. All authors have read and agreed to the published version of the manuscript.

Funding Open Access funding enabled and organized by Projekt DEAL.

\section{Compliance with ethical standards}

Conflict of interests Rajesh Devassy declares reports grants from AL Zahrawi Medical, UAE, distributors of Medtronic medical devices (Ligasure ${ }^{\mathrm{TM}}$, Sonicision ${ }^{\mathrm{TM}}$, Endo Clip ${ }^{\mathrm{TM}}$, Endo Catch ${ }^{\mathrm{TM}}$ and Veriset ${ }^{\mathrm{TM}}$ ), for educational activities conducted in UAE. The remaining authors declare not to have conflict of interests.

Ethics This report involves one human participant. The patient gave informed consent for publication. Neither IRB nor Ethical Committee permission is required for this case report.

Open Access This article is licensed under a Creative Commons Attribution 4.0 International License, which permits use, sharing, adaptation, distribution and reproduction in any medium or format, as long as you give appropriate credit to the original author(s) and the source, provide a link to the Creative Commons licence, and indicate if changes were made. The images or other third party material in this article are included in the article's Creative Commons licence, unless indicated otherwise in a credit line to the material. If material is not included in the article's Creative Commons licence and your intended use is not permitted by statutory regulation or exceeds the permitted use, you will need to obtain permission directly from the copyright holder. To view a copy of this licence, visit http://creativecommons.org/licenses/by/4.0/.

\section{References}

1. Ordulu Z, Chai H, Peng G, McDonald AG, De Nictolis M, Garcia-Fernandez E, Hardisson D, Prat J, Li P, Hui P, Oliva E, Buza N. Molecular and clinicopathologic characterization of intravenous leiomyomatosis. Mod Pathol. 2020. https://doi. org/10.1038/s41379-020-0546-8.

2. Olivia Esther, Wilbur David C, Sebire Neil J, Soslow Robert A. AFIP Atlas of Tumor Pathology. Arlington: American Registry of Pathology Press; 2020. p. 296-7.

3. Marrone G, Crinò F, Morsolini M, Caruso S, Miraglia R. Multidisciplinary approach in the management of uterine intravenous leiomyomatosis with intracardiac extension: case report and review of literature. J Radiol Case Rep. 2019;13:1-13.

4. Kim YN, Eoh KJ, Lee JY, Nam EJ, Kim S, Kim SW, Kim YT. Aberrant uterine leiomyomas with extrauterine manifestation: intravenous leiomyomatosis and benign metastasizing leiomyomas. Obstet Gynecol Sci. 2018;61:509-19.

5. Wang H, Nie P, Chen B, Hou F, Dong C, He F, Xu W. Contrastenhanced CT findings of intravenous leiomyomatosis. Clin Radiol. 2018;73:503e1-6.

Publisher's Note Springer Nature remains neutral with regard to jurisdictional claims in published maps and institutional affiliations. 


\section{About the Author}

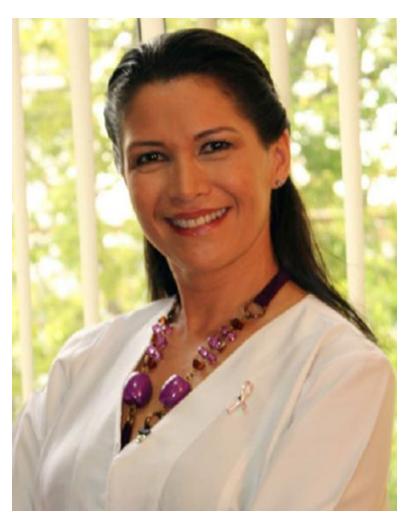

Luz Angela Torres-de la Roche is a Colombia physician. She completed her medical studies at El Bosque University of Bogotá (1987) and her specialization in obstetrics and gynecology at El Valle University, Cali (1997) and has also been trained in sexual and reproductive health at Family Health International, USA (1998). She moved to Germany on 2014, and obtained her MD degree from the Oldenburg University (2019). She has spent her career dedicated to clinical practice, research and teaching, has conducted several clinical trials and has contributed in the development of clinical guidelines and public health programs for Colombia. She has published more than 60 articles on gynecology and women's health. Currently works as a research assistant at the University Hospital for Gynaecology Pius Hospital Oldenburg, Medical Campus of the Carl von Ossietzky University, Oldenburg, Germany. 